\title{
eCommons@AKU
}

School of Nursing \& Midwifery, East Africa

Faculty of Health Sciences, East Africa

January 2015

\section{Infectious disease outbreaks and increased complexity of care}

Joan Musau

Aga Khan University, joan.musau@aku.edu

A. Baumann

McMaster University

C. Kolotylo

McMaster University

T. O'Shea

McMaster University

A. Bialachowski

St. Joseph's Healthcare

Follow this and additional works at: http://ecommons.aku.edu/eastafrica_fhs_sonam

Part of the Nursing Commons

\section{Recommended Citation}

Musau, J., Baumann, A., Kolotylo, C., O’Shea, T., Bialachowski, A. (2015). Infectious disease outbreaks and increased complexity of care. International Nursing Review, 1-8.

Available at: $\mathrm{http}: / /$ ecommons.aku.edu/eastafrica_fhs_sonam/40 


\section{Infectious disease outbreaks and increased complexity of care}

\section{J. Musau' RN, MSN, A. Baumann ${ }^{2,3}$ RN, PhD, C. Kolotylo ${ }^{4}$ RN, PhD, T. O'Shea ${ }^{5}$ MD, FRCPC, MPH \& A. Bialachowski ${ }^{6}$ RN, MS, CIC}

I Lecturer, School of Nursing and Midwifery, Nairobi, Kenya, 2 Associate Vice President Global Health, Faculty of Health Sciences, 3 Scientific Director, 4 Senior Research Associate, Nursing Health Services Research Unit, 5 Assistant Professor, Department of Medicine, Division of Infectious Diseases, McMaster University, $\mathbf{6}$ Infection Prevention and Control Manager, St. Joseph's Healthcare, Hamilton, ON, Canada

MUSAU J., BAUMANN A., KOLOTYLO C., O'SHEA T., BIALACHOWSKI A. (2015) Infectious disease outbreaks and increased complexity of care. International Nursing Review

Aim: This study examined the effects of healthcare-associated infectious disease outbreaks on nurses' work in a large acute care hospital in Ontario, Canada.

Background: The incidence of healthcare-associated infections has increased. Previous research focuses on epidemiology, healthcare systems, and the economic burden of outbreaks. Few published studies focus on the impact of outbreaks on nurses' work in acute care facilities.

Introduction: Since the severe acute respiratory syndrome epidemic in 2003, combating infectious diseases has become a key issue. Hospitals have implemented measures related to healthcare-associated infections. However, nurses experience challenges in preventing, controlling, and contending with outbreaks.

Methods: A retrospective exploratory case study approach was used. Data were collected over a 4-month period in 2012. The incidence rates of site-specific HAIs were analysed, and individual interviews were held with 23 bedside nurses and five nurse managers.

Findings: Five themes emerged from the interviews: comparison of healthcare-associated infections outbreaks; the nature of nurses' work; impact of outbreaks on patient care; innovation and quality control in clinical practice; and increased and expanded IPAC measures. The incidence rates of methicillin-resistant Staphylococcus aureus, Clostridium difficile and vancomycin-resistant enterococci at the study site decreased, but remained above provincial benchmarks. Nurses experienced workload challenges, time pressures and psychological effects stemming from outbreaks and developed various innovations in response. Patient care was also affected.

Conclusion: Nurses' work has been impacted by healthcare-associated infectious disease outbreaks. Nursing workloads should be quantified to facilitate the development of guidelines for optimum nurse-patient ratio during outbreaks.

Implications for Nursing and/or Health Policy: A strong evidence-based policy framework is required to address healthcare-associated infectious disease outbreaks. Infection prevention and control guidelines and procedures should be established provincially and nationally. An interdisciplinary approach is essential for the creation of comprehensive and innovative strategies. Nursing research has increased understanding of the

Correspondence address: Joan Musau, School of Nursing and Midwifery, Aga Khan University, P.O. Box 39340-00623, Nairobi, Kenya; Tel: 905-525-9140 ext. 22581; Fax: 905-522-5493; E-mail: joan.musau@aku.edu.

Conflict of Interest Statement: No conflict of interest is declared by the authors.

Source of Funding Statement: The study received no specific grant from any funding agency. 
implications of infectious diseases in hospitals. Building on the literature, findings from this study can be used to influence policies on the care of patients who have secondary infections. Nurse-driven protocols are important and can lead to the creation of best practice guidelines that can be implemented across settings.

Keywords: Canada, Healthcare-Associated Infections, Infection Control Policies and Procedures, Nurses, Nurses' Work, Outbreaks, Hospital Infections, Nursing Protocols

\section{Background}

Healthcare-associated infections (HAI) cause significant morbidity, mortality and economic costs (Cimiotti et al. 2012; Twomey 2013). The Centers for Disease Control and Prevention (CDC) in the United States defines HAI as 'a localized or systemic condition occurring as an adverse reaction to the presence of an infectious agent(s) or its toxin(s) that was not present on admission to the acute care facility' (CDC 2014b, p. 2).

Infectious disease has been identified as a key patient safety issue (Tella et al. 2013; Yokoe et al. 2008). The World Health Organization estimated that at any given time, at least $10 \%$ of patients ( 1.4 million people globally) suffer from the complications of infectious diseases acquired in healthcare institutions (Ducel et al. 2002). HAIs 'impose significant burdens - patient pain and suffering (including sequelae), patient-care time and resources' (Twomey 2013).

According to the Public Health Agency of Canada (2013), the number of HAIs nationwide appears to be increasing. To combat outbreaks, healthcare facilities have implemented infection prevention and control (IPAC) programmes and guidelines. Research regarding HAI has focused on epidemiology, healthcare systems and economic burden (Canadian Union of Public Employees 2009; Yokoe et al. 2008). Yet, emerging evidence demonstrates that healthcare professionals experience challenges in preventing and controlling HAI in community hospitals (Kaba 2011).

\section{Introduction}

An outbreak is defined as an increase in the number of cases above the number normally occurring in a healthcare facility over a defined period (Ducel et al. 2002). The definition is further refined in each healthcare institution and is based on the specific infecting organism as clusters of HAI occurring above a baseline (Ducel et al. 2002). In 2003, severe acute respiratory syndrome (SARS) resulted in 8096 cases and 774 deaths worldwide (Schumacher et al. 2005). Subsequent to this outbreak, government policies, reports and research studies emphasized systemic and organizational factors that contributed to the spread of SARS in healthcare facilities (Ontario Expert Panel on
SARS and Infectious Disease Control 2004; SARS Commission 2006).

Starting in 2000, four main organisms began causing HAI outbreaks in Canada and the United States: Clostridium difficile, methicillin-resistant Staphylococcus aureus (MRSA), vancomycin-resistant enterococci (VRE) and norovirus (Yokoe et al. 2008). From 2000 to 2005, C. difficile rates in the United States tripled (Gerding et al. 2008). In Canada, hospitals in British Columbia, Ontario and Quebec have experienced outbreaks of $C$. difficile resulting in patient deaths (Stastna 2013).

In 2003-2004, a C. difficile outbreak in Quebec resulted in the death of 100 patients and led to massive IPAC efforts (Eggertson 2005). A new strain of $C$. difficile (NAP1) identified during the outbreak was associated with high levels of morbidity and mortality (Loo et al. 2005). Over a 20-month period starting in 2006, more than 200 patients in an Ontario hospital contracted C. difficile, 91 patients died (Metroland News Service 2014). Studies on nurses' work generally focus on HAI occurrence and compliance with IPAC policies and procedures (Al-Rawajfah et al. 2013; Erasmus et al. 2010). The results of this study, conducted over a 4-month period in 2012, demonstrate how nurses' work in one acute care hospital in Ontario changed because of HAI outbreaks.

\section{Methods}

\section{Design}

A retrospective exploratory case study design was used in this study. One of the strengths of case study design is the application of multiple evidence sources (Yin 2009). In this study, secondary data analysis of infection rates, individual interviews and document analysis were used.

\section{Sample}

Purposive sampling was used to select bedside nurses and nurse managers from surgical, medical, nephrology and psychiatric units at the acute care hospital. The study included participants of either gender and of any age who were fluent in English and willing to take part. Participants were employed by the acute 
care hospital at the time of the study and were involved in at least one HAI outbreak. All nurse managers at the site were sent an introductory email describing the purpose and objectives of the study, potential participants required, and proposed data collection methods. Twenty-eight participants were recruited and individual interviews were scheduled.

\section{Ethical considerations}

This study was approved by ethics committees at an academic institution and the acute care hospital. Written informed consent was obtained from all participants.

\section{Data collection}

The IPAC Department at the hospital provided cumulative data on infectious disease incidence; mandatory reportable data on VRE, C. difficile and MRSA incidence; and site-specific data on HAI outbreaks from 2008 to 2012. Participant interviews were conducted from September to December of 2012. Interviews were held privately, audiotaped and completed in 30-60 min. To focus the interviews on HAI outbreaks and the effects on nurses' work, a researcher-developed semi-structured interview guide with probes was used. Unbiased questioning was employed to minimize partiality and increase objectivity.

Document analysis is used in combination with other research methods to triangulate data in qualitative research (Bowen 2009; Miller \& Alvarado 2005), provide data on context, track relevant changes (Bowen 2009), and corroborate information from other sources (Yin 2009). In this study, the acute care hospital policies and protocols specific to HAI and outbreaks were reviewed in depth and analysed.

\section{Data analysis}

Aggregated anonymous cumulative data were used to generate descriptive statistics for MRSA, VRE and C. difficile incidence rates for the study period. The rates were analysed and graphed per 1000 patient days.

\section{Participant interviews}

The interview tapes were transcribed verbatim and imported into NVivo 10 for Microsoft Windows (2012). Data reduction began with open coding. Initially, three transcripts were selected at random and coded independently by two of the researchers to determine the reliability of the coding process. Consensus on the coding choices was reached through discussion. The researchers used constant comparison to interpret the structure and relationships of the concepts that emerged from the data (Patton 2002). Data were collected until thematic saturation occurred (Miles \& Huberman 1994). All members of the research team agreed on the final themes and conclusions made based on the data.

\section{Document analysis}

IPAC policies and protocols specific to HAI outbreaks were examined. Findings from the documentary sources were used to develop converging or diverging lines of inquiry, which promotes the corroboration of data sources and strengthens the overall findings by means of data triangulation (Bowen 2009; Miller \& Alvarado 2005; Yin 2009).

\section{Findings}

\section{Incidence rates of infectious diseases}

As shown in Fig. 1, VRE had the highest incident rate, followed by MRSA, and C. difficile during the study period. The incidence of both MRSA and C. difficile rose in the following years, peaked, and then decreased in 2012 to a rate higher than in 2008. C. difficile peaked in 2010 and MRSA peaked in 2011. The VRE incident rate was the highest in 2008 and steadily decreased to a much lower rate in 2012, with a slight increase in 2011. C. difficile is the greatest concern at the study site because it has caused two facility-wide outbreaks (November 2010 to February 2011 and summer 2012). The site has also experienced eight unit-based VRE outbreaks (February to November 2011) and four unit-based MRSA outbreaks (July 2011 to August 2012).

\section{Demographic data}

Demographic data were collected from 23 bedside nurses and five nurse managers. All were women and their mean age was

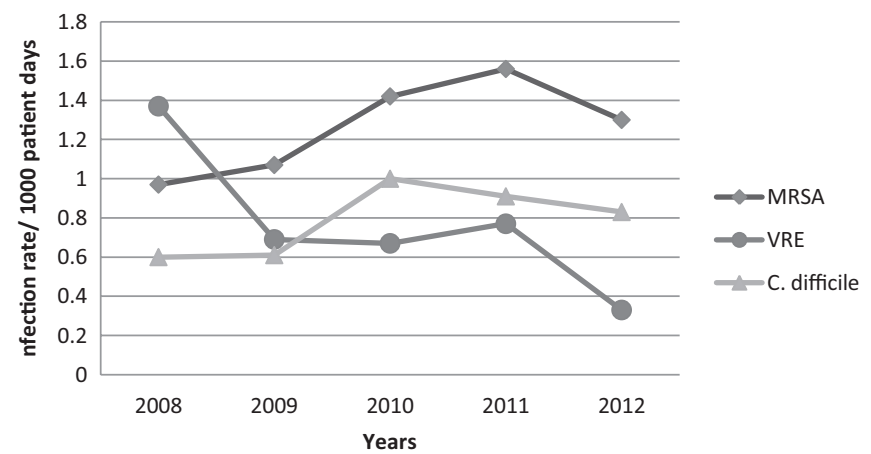

Fig. 1 Incidence of methicillin-resistant Staphylococcus aureus and vancomycin-resistant enterococci colonizations and infections, and Clostridium difficile infections (2008-2012). 
40.3 years [standard deviation $(\mathrm{SD})=10.3, \mathrm{Mdn}=43.0$ years] . Twenty-six participants $(92.9 \%)$ were employed full-time and two $(7.1 \%)$ were employed part-time. The mean number of years of employment at the site was $10.2(\mathrm{SD}=8.5)$.

\section{Participant interviews}

Five themes emerged from the semi-structured interviews and they are discussed in detail later.

\section{Theme 1: comparison of HAI outbreaks}

Participants discussed the facility-wide and unit outbreaks. They indicated that the unit-based outbreaks were usually caused by MRSA, VRE and norovirus. These outbreaks drew little media attention and IPAC measures were localized to the unit. In contrast, the two facility-wide outbreaks were caused by C. difficile and resulted in increased scrutiny and attention from the media, hospital administration, and public and required broad implementation of IPAC measures. They also caused emotional stress. Some healthcare team members felt they were held responsible for the outbreaks, which made them defensive with friends, relatives, patients and colleagues. A bedside nurse remarked, 'We get blamed if the infection spreads, but we are not the only people responsible for providing care to patients.'

Participants reported that the facility and staff were better prepared for the enormity of the IPAC task during the second outbreak than during the first. An external IPAC audit was conducted after the first outbreak to evaluate the cause and contributing factors. Numerous policies and practices were created or modified as a result. For example, to decrease the transmission of infectious diseases between patients, patient-dedicated equipment was used and patients were placed in isolation in private rooms. Furthermore, requirements for the use of personal protective equipment $(\mathrm{PPE})$ and hand hygiene $(\mathrm{HH})$ compliance rates were increased for all healthcare professionals.

Most participants credited the new/modified IPAC measures for the second outbreak being shorter and less severe than the first. One nurse manager commented, 'After the 2010-2011 C. difficile outbreak, there were consistent processes and improved communication among hospital departments and the senior management team.' Guidelines for the cleaning and decontamination of equipment and physical plant were also enacted.

Theme 2: the nature of nurses' work

Bedside nurses emphasized that a new normal exists for nursing care because of the proliferation of infectious diseases and the number of patients requiring isolation precautions. A bedside nurse said, 'I honestly don't remember having a patient with C. difficile infection in my first three years of nursing.' The nurses described a supplementary level of care beyond the usual level and increased activities resulting from IPAC measures. For example, more frequent screening and swabbing of patients on admission to the hospital and during their stay. One nurse observed, 'Everyone that walks into our unit for admission is screened for MRSA and VRE infection. We never used to do that.'

Participants indicated that mandated HAI prevention education increased during outbreaks. In-service sessions were tailored to reinforce compliance with IPAC policies and practices. Participants were proactive in trying to prevent and control the transmission of infectious diseases. One nurse remarked, 'We spend a lot of time educating patients' families and visitors about the necessity of infection control, patient isolation, and the use of PPE.'

Participants also acknowledged that certain aspects of their work, such as documentation, increased during outbreaks, but the nurse-patient ratio did not change in response. The normal nurse-patient ratios are one nurse to five patients on day shifts and one nurse to six or seven patients on night shifts. This created challenges during outbreaks. Because of the pathophysiology of C. difficile, infected patients required increased assistance with toileting. Each time a nurse entered and exited an isolation room, an additional 3-4 min was required for IPAC procedures.

Participants indicated that, on average, an hour of every shift was spent on HH and PPE. However, when patients had loose bowel movements, nurses spent extra time cleaning the area, dressing patients in fresh gowns, and putting on and removing PPE. In these instances, $\mathrm{HH}$ and PPE took from 10 to $30 \mathrm{~min}$ per patient. This averages out to $4 \mathrm{~h}$ per 12 -h shift, a significant amount of time.

Theme 3: impact of outbreaks on patient care

Nurses expressed concern that the provision of general nursing care was not completed in a timely manner or at all, particularly when their workloads were very heavy. The nurses cared for isolated patients for long periods. One nurse commented, 'sometimes I am in a room for $45 \mathrm{~min}$, and that is $45 \mathrm{~min}$ that I am not there for the other four patients to whom I am assigned or a family member that had a question to ask.' Another nurse added, 'During outbreaks, we are required to multitask more. Sometimes the care we provide to patients is affected.' Some nurses contended that heightened demands and increased workloads led to burnout, which resulted in increased sick time and potential for errors (e.g. medication errors).

Participants also reported they were not meeting the psychosocial needs of patients, especially patients in isolation. Bedside nurses mentioned rushing through care for one patient so they 
could begin care for the next patient. This allowed little time to establish therapeutic nurse-patient relationships. One nurse said, 'We limit the number of times we enter isolation rooms because of the PPE requirement. Therefore, isolated patients may feel lonely and neglected.' The nurses observed that isolated patients have fewer visitors and attributed this to the visitors' fears of acquiring infectious diseases. This fear extended to the nurses as well. Participants were afraid of acquiring infectious diseases or transmitting them to family members and perceived that the risk of doing so was higher during outbreaks.

Theme 4: innovation and quality control in clinical practice

To improve patient care and strengthen quality control, various innovations and interventions were operationalized after the first facility-wide outbreak. For example, nurse servers for storing PPE supplies were built into the wall outside patients' rooms. The servers are well stocked to increase efficiency and protected by doors to minimize contamination. The participants expressed high satisfaction with the servers.

An HAI prevention audit tool was created to evaluate factors contributing to the transmission of infectious diseases. It is used to examine hospital units reporting new HAI cases. The tool was formulated as a checklist based on an accepted facility theory, the five $\mathrm{C}$ model method of communication. The five Cs in the model represent clean equipment, clean environment, clean hands, clear communication, and consistent processes. A screensaver was installed on all computer monitors to remind staff about the five Cs.

During outbreaks, whiteboards are mounted on the walls in unit hallways and at all nursing stations. They display coloured graphs of the rates of infection and $\mathrm{HH}$ compliance in each unit. The boards are updated daily by the nurse managers. To further facilitate the exchange of key information regarding infectious diseases, staff huddles are held twice daily during outbreaks. A nurse explained that during these 15 to $20 \mathrm{~min}$ meetings, infection prevention and control practices are reiterated and quick questions about infectious diseases can be asked and answered.'

Participants described interventions specifically targeting C. difficile. In a 6-month pilot, a specially designated infection control professional tracked patients with $C$. difficile in the hospital and the community upon discharge and acted in an educational capacity with the patients and their families. Multidisciplinary care plans were also used to manage patients and reduce the risk of transmission.

To reinforce its commitment to IPAC, the hospital placed hand sanitizers in and outside all patient rooms, beside patient beds and at regular intervals in the hallways throughout the hospital. A taped message advised all people entering and exiting the hospital to use the sanitizers. Furthermore, the hospital purchased equipment for dedicated use in the isolation rooms. A 'tagging and bagging' system was instituted to identify cleaned and disinfected equipment.

Other efforts to reduce the transmission of infectious disease included changing washbasins weekly for non-isolated patients and daily for isolated patients. This practice is particularly important because $C$. difficile spores cannot be eliminated by regular cleaning procedures. To reduce the incidence and colonization of VRE and MRSA, it was mandated that patients with these infections use soap containing chlorhexidine gluconate for a 14-day period.

\section{Theme 5: increased and expanded IPAC measures}

The participants noted that the hospital had an $\mathrm{HH}$ policy that had been in effect and developed over the years. After the first facility-wide outbreak, expectations for $\mathrm{HH}$ compliance rates for all healthcare professionals increased from 60 to $90 \%$. Diamond rings, nail polish and artificial nails are no longer permitted in the clinical areas.

Additional policies and practices were enforced regarding de-cluttering of the hospital units, restricting food in clinical areas and implementing the 'no visitors policy.' Historically, the hospital visitor policy allowed patients to have two visitors at a time. However, during an outbreak, infection control professionals and nurse managers could jointly decide to implement the no visitors policy to restrict traffic in the units.

The in-hospital patient transfer policy was also reviewed and changed. Patients are now transferred primarily for clinical reasons. Unless necessary, patients are not transferred in hospital beds, they are transferred by stretcher or wheelchair and the furniture in their rooms is no longer transferred with them. The practice of cohorting patients with the same infectious diseases was also revised. Cohorting is defined as,

The assignment of a geographic area such as a room or a patient care area to two or more clients/patients/residents who are either colonized or infected with the same microorganism, with staffing assignments restricted to the cohorted group of patients (Provincial Infectious Diseases Advisory Committee 2012, p. viii).

Patients with infectious diseases are now isolated in private rooms with dedicated bathrooms.

\section{Discussion}

Findings from this study demonstrate that the work of nurses at the site has changed and become more complex because of the greater incidence of HAI. Participants agreed that the number of outbreaks and the number of isolated in-hospital patients 
had increased. A contributing factor to higher infectious disease incidence rates was that patients were colonized with community-acquired infectious diseases prior to their admission, which added to the overall bioburden in the facility (gata et al. 2009).

A point prevalence survey of acute care hospitals in Europe found that 'twenty-three percent of HAIs $(n=3503)$ were present on admission' (European Centre for Disease Prevention and Control 2013, p. 4). gata et al. (2009) note the prevalence of community-acquired MRSA is increasing and will become the dominant strain in the next decade. Just as the increase in community-acquired $C$. difficile in Ontario translated into more hospital cases than was anticipated, community-acquired MRSA may do the same.

In a recent news article, the Director of the Royal College of Nursing stated, 'Infection prevention and control are key to patient safety and need to be prioritised by every health service organisation' (British Broadcasting Corporation 2014). In their multistate survey, Magill et al. (2014) concluded, 'public health surveillance and prevention activities should continue to address $C$. difficile infections ... [and] consideration should be given to expanding surveillance and prevention activities to include other health care-associated infections (p. 1198).

C. difficile and MRSA are ranked among the 10 highest burden pathogens (Kwong et al. 2012), and there is mounting concern in the healthcare sector regarding emerging infectious diseases such as carbapenem-resistant Enterobacteriaceae and Middle-East respiratory syndrome (CDC 2014a,c). The Canadian Nosocomial Infection Surveillance Program closely monitors C. difficile, MRSA, and VRE (Public Health Agency of Canada 2013).

Participants specifically cited $C$. difficile outbreaks as affecting their work. As both a responsive and precautionary IPAC measure, the study site may consider reinstituting the $C$. difficile infection control professional to follow up with C. difficile patients who have been discharged from the hospital into the community. The provincial government mandated the public reporting of in-hospital patients with infectious diseases in 2008 (Ontario Ministry of Health and Long-Term Care 2013).

Nurses at the study site had to adjust to a new normal precipitated by outbreaks and strove to incorporate both changes to and an increased number of IPAC policies and practices. The nurses' work was impacted by the cumulative effect of additional time spent on IPAC measures, the increased number of patients requiring isolation precautions, the greater complexity of patient care and the static nurse-patient ratios. The literature demonstrates that the risk of acquiring HAI is associated with excessive workloads and insufficient staffing (Daud-Gallotti et al. 2012). According to Cimiotti et al. (2012):
Health care facilities can improve nurse staffing and other elements of the care environment and alleviate job-related burnout in nurses at a much lower cost than those associated with health care-associated infections. By reducing nurse burnout, we can improve the well being of nurses while improving the quality of patient care (p. 5).

Participants highlighted the issue of patient care and indicated that the psychosocial needs of patients were not being met during outbreaks, especially patients in isolation. This finding is aligned with Kalisch's (2006) concept of 'missed care,' which is defined as 'any aspect of required patient care that is omitted (either in part or in whole) or delayed' (Kalisch et al. 2009, p. 4). This includes interventions such as patient education, emotional support, surveillance, and hygiene (Kalisch 2006). Patients in isolation often feel stigmatized and may perceive that the quality of care they receive is adversely affected by their diagnosis (Madeo 2003; Mozzillo et al. 2010).

\section{Study limitations}

The study was confined to one large acute care hospital in Ontario, Canada. Therefore, the findings may not be generalizable to other settings. Because purposive sampling was used to select participants who were accessible, experienced with the phenomenon of interest, and willing to participate, sampling may have been subject to volunteer bias. The nurses and nurse managers who participated in the study may be different from the general population of nurses and nurse managers working in other acute care hospitals. Thus, the study findings may not be generalizable to other nurses.

\section{Conclusions}

The study demonstrated that nurses' work in one acute care hospital has been impacted by HAI and outbreaks. In response, patient care innovations were created, including increased communication strategies and targeted physical adaptations, which became part of the organizational policy framework. Nursing workloads should be quantified to facilitate the development of guidelines for optimum nurse-patient ratio during outbreaks. Additional longitudinal studies should examine factors that might contribute to the increase of specific HAI.

\section{Implications for nursing and/or health policy}

A strong evidence-based policy framework is required to address HAI and outbreaks from existing and emerging diseases. IPAC guidelines and procedures should be established provincially and nationally. An interdisciplinary approach is essential for the creation of comprehensive and innovative strategies to decrease the incidence of HAI and shorten the length of 
outbreaks. Nursing research has increased understanding of the implications of infectious diseases in hospitals. Building on the literature, findings from this study can be used to influence policies on the care of patients who have secondary infections. Nurse-driven protocols are important and can lead to the creation of best practice guidelines that can be implemented across settings.

\section{Acknowledgements}

The authors would like to thank the participants for their contributions to the study.

\section{Author contributions}

JM was involved in all the processes of the study (recruitment, data collection and analysis), writing and submitting the paper, and addressing the comments of the editor and reviewers in the revised draft of paper. $\mathrm{ABa}$ was involved in all the processes of the study (recruitment, data collection and analysis) and writing the paper. CK was involved in all the processes of the study (recruitment, data collection and analysis) and writing the paper. TOS was involved in data analysis and writing the paper. $\mathrm{ABi}$ was involved in recruitment, data collection and writing the paper.

\section{References}

Al-Rawajfah, O.M., et al. (2013) Compliance of Jordanian registered nurses with infection control guidelines: a national population-based study. American Journal of Infection Control, 41 (11), 1065-1068.

Bowen, G.A. (2009) Document analysis as a qualitative research method. Qualitative Research Journal, 9 (2), 27-40.

British Broadcasting Corporation (2014) Hospital infection rates must come down, says watchdog. BBC News, online 16 April 2014. Available at: http://www.bbc.com/news/health-27046990 (accessed 22 April 2014).

Canadian Union of Public Employees (2009) Healthcare associated infections: a backgrounder. Available at: http://cupe.ca/updir/healthcareassociated-infections-cupe-backgrounder.pdf (accessed 20 November 2013).

Centers for Disease Control and Prevention. (2014a) Healthcare-associated Infections (HAIs): Carbapenem-resistant Enterobacteriaceae in Healthcare Settings. Available at: http://www.cdc.gov/hai/organisms/cre/ (accessed 15 May 2014).

Centers for Disease Control and Prevention. (2014b) Identifying Healthcare-associated Infections (HAI) in NHSN: Present on Admission (POA) Infections. Available at: http://www.cdc.gov/nhsn/pdfs/pscmanual/ 2psc_identifyinghais_nhsncurrent.pdf (accessed 22 April 2014).

Centers for Disease Control and Prevention. (2014c) Middle East Respiratory Syndrome (MERS). Available at: http://www.cdc.gov/ CORONAVIRUS/MERS/INDEX.HTML (accessed 15 May 2014). Cimiotti, J.P., Aiken, L.H., Sloane, D.M. \& Wu, E.S. (2012) Nurse staffing, burnout, and health care-associated infection. American Journal of Infection Control, 40 (6), 486-490.
Daud-Gallotti, R.M., et al. (2012) Nursing workload as a risk factor for healthcare associated infections in ICU: a prospective study. PLoS ONE, 7 (12), e52342.

Ducel, G., Fabry, J. \& Nicolle, L., eds (2002) Prevention of Hospital Acquired Infections: A Practical Guide, 2nd edn. World Health Organization, Malta. World Health Organization, Department of Communicable Disease, Surveillance and Response.

Eggertson, L. (2005) C. difficile may have killed 2000 in Quebec: study. Canadian Medical Association Journal, 173 (9), 1020-1021.

Erasmus, V., et al. (2010) Systematic review of studies on compliance with hand hygiene guidelines in hospital care. Infection Control and Hospital Epidemiology, 31 (3), 283-294.

European Centre for Disease Prevention and Control (2013) Point Prevalence Survey of Healthcare-Associated Infections and Antimicrobial Use in European Acute Care Hospitals. ECDC, Stockholm.

D'Agata, E., et al. (2009) Modeling the invasion of community-acquired methicillin-resistant Staphylococcus aureus into hospitals. Clinical Infectious Diseases, 48 (3), 274-284.

Gerding, D.N., Muto, C.A. \& Owens, R.C. Jr (2008) Measures to control and prevent Clostridium difficile infection. Clinical Infectious Diseases, 46 (Suppl. 1), S43-S49.

Kaba, A. (2011) The Changing Nature of the Health Professionals' Work: The Impact of Infectious Diseases. Master's Thesis, McMaster University, Hamilton, Canada.

Kalisch, B.J. (2006) Missed nursing care: a qualitative study. Journal of Nursing Care Quality, 21 (4), 306-313.

Kalisch, B.J., Landstrom, G. \& Williams, R.A. (2009) Missed nursing care: errors of omission. Nursing Outlook, 57 (1), 3-9.

Kwong, J.C., et al. (2012) The impact of infection on population health: results of the Ontario burden of infectious diseases study. PLoS ONE, 7, e44103.

Loo, V.G., et al. (2005) A predominantly clonal multi-institutional outbreak of Clostridium difficile-associated diarrhea with high morbidity and mortality. New England Journal of Medicine, 353, 2442-2449.

Madeo, M. (2003) The psychological impact of isolation. Nursing Times, 99 (7), 54-55.

Magill, S.S., et al. (2014) Multistate point-prevalence survey of health careassociated infections. New England Journal of Medicine, 370 (13), 11981208.

Metroland News Service. (2014) Hamilton General may declare C. diff outbreak. Available at: http://metronews.ca/news/hamilton/923815/ hamilton-general-may-declare-c-diff-outbreak/ (accessed 30 January 2014).

Miles, M. \& Huberman, M. (1994) Qualitative Data Analysis. Sage, Thousand Oaks, CA.

Miller, F.A. \& Alvarado, K. (2005) Incorporating documents into qualitative nursing research. Journal of Nursing Scholarship, 37 (4), 348353.

Mozzillo, K.L., Ortiz, N. \& Miller, L.G. (2010) Patients with MethicillinResistant Staphylococcus aureus (MRSA) infection - 21st century lepers. Journal of Hospital Infection, 75 (2), 132-134.

NVivo 10 for Microsoft Windows (2012) Software, version 10.0. QSR International Pty Ltd, Doncaster, Victoria, Australia. 
Ontario Expert Panel on SARS and Infectious Disease Control (2004) For the Public's Health: A Plan for Action. Government of Ontario, Toronto, Canada.

Ontario Ministry of Health and Long-Term Care (2013) Infectious Diseases Protocol, 2013. Ontario Public Health Standards. Ministry of Health and Long-Term Care, Toronto, Canada. Available at: http:// www.health.gov.on.ca/en/pro/programs/publichealth/oph_standards/ docs/infectious_diseases.pdf (accessed 24 April 2014).

Patton, M.Q. (2002) Qualitative Research \& Evaluation Methods, 3rd edn. Sage, Thousand Oaks, CA.

Provincial Infectious Diseases Advisory Committee (2012) Routine Practices and Additional Precautions: In All Health Care Settings, 3rd edn. Ontario Agency for Health Protection and Promotion. Queen's Printer for Ontario, Toronto, Canada.

Public Health Agency of Canada (2013) The Chief Public Health Officer's Report on the State of Public Health in Canada, 2013: Infectious Disease The Never-ending Threat. Available at: http://www.phac-aspc.gc.ca/ cphorsphc-respcacsp/2013/infections-eng.php\#footnote1 (accessed 24 April 2014).

SARS Commission (2006) SARS Commission Executive Summary: Volume One - Spring of Fear. Ontario Ministry of Health and Long-Term Care, Toronto, Canada.
Schumacher, K.L., et al. (2005) Qualitative research contribution to a randomized clinical trial. Research in Nursing \& Health, 28 (3), 268-280.

Stastna, K. (2013) Exclusive: Dirty Hospital Rooms A Top Concern for Canadians. Available at: http://www.cbc.ca/news/health/dirty-hospitalrooms-a-top-concern-for-canadians-1.1321668 (accessed 3 May 2014).

Tella, S., et al. (2013) What do nursing students learn about patient safety? An integrative literature review. Journal of Nursing Education, 53 (1), $1-15$.

Twomey, C. (2013) The Surgical Healthcare-Associated Infection: Uncovering the Truth about Surgical Irrigation. ICT: Infection Control Today. Available at: http://www.infectioncontroltoday.com/articles/2013/ 07/the-surgical-healthcareassociated-infection-uncovering-the-truthabout-surgical-irrigation.aspx (accessed 21 April 2014).

Yin, R.K. (2009) Case Study Research: Design and Methods, 4th edn. Sage, Thousand Oaks, CA.

Yokoe, D.S., et al. (2008) A compendium of strategies to prevent healthcare-associated infections in acute care hospitals. Infection Control and Hospital Epidemiology, 29 (Suppl. 1), S12-S21. 